$$
\begin{array}{r}
\text { DOE/ER/ } 40272 \cdots-268 \\
\text { Institute for Fundamental Theory Preprint UFIFT }- \text { HEP-97-3 }
\end{array}
$$

\title{
Spin Dependent Drell-Yan beyond Leading Order: Non-Singlet Virtual corrections to $O\left(\alpha_{s}^{2}\right)$
}

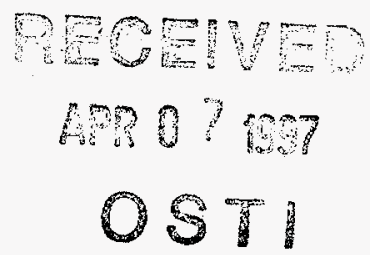

\author{
Sanghyeon Chang ${ }^{1}$, Claudio Coriano ${ }^{2}$ and R. D. Field ${ }^{3}$ \\ ${ }^{1}$ Institute for Fundamental Theory, Department of Physics, \\ University of Florida, Gainesville, FL 32611, USA
}

\begin{abstract}
We present parton-level analytical results for the next-to-leading order nonsinglet virtual corrections to the Drell-Yan differential cross-section. The dependence of the differential cross section on the helicity of the initial state partons is shown explicitly (the spins of the final state partons are summed). The calculation is implemented in dimensional regularization within the $\overline{M S}$ scheme and with the t'Hooft Veltman prescriptions for the n-dimensional $\gamma_{5}$. Both the polarized initial state and the unpolarized cross sections can be obtained from our result. Our unpolarized cross section agrees with the previous result of Ellis, Martinelli and Petronzio in the non-singlet sector.
\end{abstract}

\footnotetext{
${ }^{1}$ E-mail address: schang@phys.uf.edu

${ }^{2}$ E-mail address: coriano@phys.ufl.edu

${ }^{3} \mathrm{E}$-mail address: rfield@phys.uf.edu
}

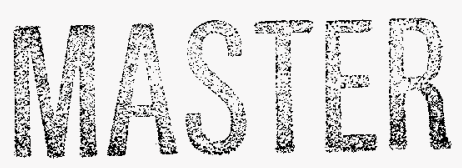

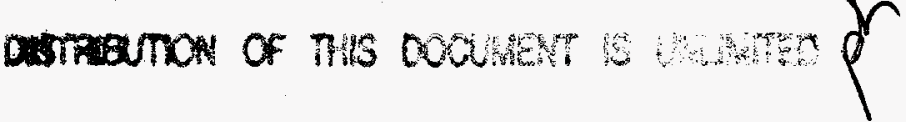




\section{DISCLAIMER}

This report was prepared as an account of work sponsored by an agency of the United States Government. Neither the United States Government nor any agency thereof, nor any of their employees, make any warranty, express or implied, or assumes any legal liabiijty or responsibility for the accuracy, completeness, or usefulness of any information, apparatus, product, or process disclosed, or represents that its use would not infringe privately owned rights. Reference herein to any specific commercial product, process, or service by trade name, trademark, manufacturer, or otherwise does not necessarily constitute or imply its endorsement, recommendation, or favoring by the United States Government or any agency thereof. The views and opinions of authors expressed herein do not necessarily state or reflect those of the United States Government or any agency thereof. 


\section{DISCLAIMER}

Portions of this document may be illegible in electronic image products. Images are produced from the best available original document. 


\section{Introduction}

Spin physics has emerged in the last few years as one of the most active fields of perturbative QCD. Given the planned experiments at RHIC and HERA, it is likely that such interest will continue to grow. Among the interesting processes which are planned to be investigated at hadron colliders such as RHIC are the Drell-Yan production of muon pairs and two-jet production in polarized proton-proton collisions [1]. The difficulty of calculating spin dependence in next-to-leading order QCD is notorious and therefore only $O\left(\alpha_{s}\right)$ results have been presented in the literature.

The difficulty in the calculations comes from the presence of chiral fermions or gluons of fixed helicity in the initial state which renders the study of the spin dependencies extremely time consuming. The analysis of the infrared structure of the cross section in perturbation theory is also much more complex than the unpolarized case. If dimensional regularization is used to control both the infrared and ultraviolet singularities of the amplitudes, then the presence of chiral projector for the fermions requires a suitable definition of $\gamma_{5}$ in $n$-dimension. In this work we present results for the $O\left(\alpha_{s}^{2}\right)$ virtual corrections to the $q \bar{q}$ annihilation channel for the classical Drell-Yan process. This is an important ingredient to the overall non-singlet differential cross section, $d \sigma / d Q^{2} d t d u$, and for the total cross section $\sigma\left(Q^{2}\right)$, where $Q^{2}$ is the invariant mass of the photon. The differential cross section is used to predict the transverse momentum distribution of the photon in the process $p p \rightarrow \gamma^{*}+X$ with polarized beams.

It has been observed [8] that Drell-Yan production of charged lepton pairs is a case where transverse and longitudinal asymmetries are comparable. To leading order, the ratio between the transverse and the longitudinal asymmetries $R \equiv A_{T T} / A_{L L}$ is estimated to be of order 1 . This result is due to the fact that the gluons don't couple to transverse asymmetries and to order $\alpha_{s}$ only the quark-antiquark channel contribute. Furthermore, Drell-Yan is one of the possible avenues to obtain information about the twist-2 transversity distribution, which can be related to the ratio between transverse and longitudinal asymmetries.

It is important to study the polarized Drell-Yan process, including its factorization and renormalization scale dependence by going to next-to-leading order. The Drell-Yan process has been always considered a possible way to disentangle possible new gauge interactions. The inclusion of polarization in the initial states can be a useful tool to investigate such effects, and other effects, such as compositeness and supersymmetry (see [2] and references therein). The presence of a massive photon in the final state makes an analytic calculation much more involved than, for instance, two other recent similar studies [4]. Although the non-singlet contribution to the process can only give information about the polarized quark distributions, it is the most lengthy Drell-Yan subprocess. We present the order $\alpha_{s}^{2}$ virtual corrections in this paper. Work on the initial state helicity dependence of the real corrections is close to completion [12] and will follow shortly. 


\section{General Structure}

The lowest order (non-singlet) contributions to the large transverse momentum production of virtual photons (see Fig. 1) with invariant mass $q^{2}=Q^{2}$ arise from the two, $q+\bar{q} \rightarrow \gamma^{*}+g$, "Born" amplitudes shown in Fig. 3. We refer to these two diagrams as the direct and the exchange (or crossed) amplitudes, respectively. In Fig. 2 we have generically illustrated the expansion of the amplitude which appears in $d \sigma / d Q^{2}$ up to order $\alpha_{s}$. Notice that the quark form factor contributions represented in Fig. 3 (and the related $O\left(\alpha_{s}^{2}\right)$ corrections, not included in the picture) do not appear in the study of the cross section, $d \sigma / d^{2} q_{t} d y$, for transverse momentum, $q_{t}$, greater than zero. The two-loop $O\left(\alpha_{s}^{2}\right)$ corrections to the quark form factor can be added in the study of the total cross section, $d \sigma / d Q^{2}$, by interfering the 2-loop on-shell quark form factor of ref. [11] with the lowest order $q \bar{q} \gamma$ annihilation channel and by using helicity projectors for the initial quark states.

We calculate the spin dependence of the cross section by using the helicity projectors, $P_{ \pm}=\frac{1}{2}\left(1 \pm \gamma_{5}\right)$, which project out the helicity states of an initial state quark and antiquark, respectively, as follows:

$$
u\left(p_{1}, h_{1}\right)=\frac{1}{2}\left(1+h_{1} \gamma_{5}\right) u(p), \bar{v}\left(p_{2}, h_{2}\right)=\frac{1}{2} \bar{v}\left(p_{2}\right)\left(1-h_{2} \gamma_{5}\right),
$$

where $h_{1}= \pm 1$ corresponds to quark helicity $\pm \frac{1}{2}$, and $h_{2}= \pm 1$ corresponds to antiquark helicity $\mp \frac{1}{2}$.

The squares of the direct amplitude $L_{1}$ and exchange amplitude $L_{2}$ in Fig. 3 in $N=4-2 \epsilon$ dimensions are given by

$$
\begin{aligned}
& M_{d d}\left(h_{1}, h_{2}\right)=e_{f}^{2} g^{2} g_{s}^{2} \frac{C_{F}}{N_{c}} \frac{2 u}{t}\left((1-\epsilon)^{2}+h_{1} h_{2}(1+\epsilon)^{2}\right), \\
& M_{c c}\left(h_{1}, h_{2}\right)=e_{f}^{2} g^{2} g_{s}^{2} \frac{C_{F}}{N_{c}} \frac{2 t}{u}\left((1-\epsilon)^{2}+h_{1} h_{2}(1+\epsilon)^{2}\right) .
\end{aligned}
$$

where $\alpha=g^{2} / 4 \pi$ is the fine structure constant, and $e_{f}$ the charge of the quark. The quantity $C_{F} / N_{c}$ is the color factor, and $\alpha_{s}=g_{s}^{2} / 4 \pi$ is the QCD strong coupling constant. The interference term is more complicated and is given by,

$$
\begin{aligned}
& 2 M_{d c}\left(h_{1}, h_{2}\right)= \\
& e_{f}^{2} g^{2} g_{s}^{2} \frac{C_{F}}{N_{c}} \frac{4}{t u}\left[(1-\epsilon)\left(Q^{2} s-\epsilon t u\right)+h_{1} h_{2}(1+\epsilon)\left(Q^{2} s+\epsilon t u\right)+2 h_{1} h_{2} \epsilon t u\right] .
\end{aligned}
$$

The sum of the two Born amplitudes squared is

$$
\begin{aligned}
& \left|M_{B}\left(h_{1}, h_{2}\right)\right|^{2}=\left|M_{B}(h)\right|^{2}=M_{d d}(h)+2 M_{d c}(h)+M_{c c}(h) \\
& =e_{f}^{2} g^{2} g_{s}^{2} \frac{C_{F}}{N_{c}} \frac{2}{t u}\left[(1-\epsilon)\left(2 Q^{2} s+(1-\epsilon)\left(t^{2}+u^{2}\right)-2 \epsilon t u\right)\right. \\
& \left.+h(1+\epsilon)\left(2 Q^{2} s+(1+\epsilon)\left(t^{2}+u^{2}\right)+2 \epsilon t u\right)+4 h \epsilon t u\right],
\end{aligned}
$$


and depends only on the product $h=h_{1} h_{2}$.

To any order in perturbation theory we can write,

$$
\left|M\left(h_{1}, h_{2}\right)\right|^{2}=|M(h)|^{2}=(1+h)|\bar{M}|^{2}-h\left|M_{++}\right|^{2}=|\bar{M}|^{2}-h \Delta|M|^{2},
$$

where $h=h_{1} h_{2}$ and where

$$
|\bar{M}|^{2}=\frac{1}{4}\left|\sum_{h_{1}, h_{2}} M\left(h_{1}, h_{2}\right)\right|^{2}=\frac{1}{4} \sum_{h_{1}, h_{2}}\left|M\left(h_{1}, h_{2}\right)\right|^{2},
$$

is the spin averaged (unpolarized) amplitude squared. Furthermore,

$$
\left|M_{--}\right|^{2}=\left|M_{++}\right|^{2} \text { and }\left|M_{-+}\right|^{2}=\left|M_{+-}\right|^{2},
$$

so that

$$
|\bar{M}|^{2}=\frac{1}{2}\left(\left|M_{++}\right|^{2}+\left|M_{+-}\right|^{2}\right) .
$$

The spin asymmetry, $\Delta|M|^{2}$, is defined according to

$$
\left|M_{\Delta}\right|^{2}=\frac{1}{2}\left(\left|M_{++}\right|^{2}-\left|M_{+-}\right|^{2}\right)=\left|M_{++}\right|^{2}-|\bar{M}|^{2} .
$$

The spin averaged (unpolarized) amplitude squared is determined from $|M(h)|^{2}$ by setting $h=0$ and $-\Delta|M|^{2}$ is the coefficient of $h$. For the Born term, this results in

$$
|\bar{M}|^{2}=e_{f}^{2} g^{2} g_{s}^{2} \frac{C_{F}}{N_{c}} \frac{2}{t u}\left[(1-\epsilon)\left(2 Q^{2} s+(1-\epsilon)\left(t^{2}+u^{2}\right)-2 \epsilon t u\right)\right],
$$

and

$$
\Delta|M|^{2}=-e_{f}^{2} g^{2} g_{s}^{2} \frac{C_{F}}{N_{c}} \frac{2}{t u}\left[(1+\epsilon)\left(2 Q^{2} s+(1+\epsilon)\left(t^{2}+u^{2}\right)+2 \epsilon t u\right)+4 \epsilon t u\right] .
$$

Adding these two terms yields

$$
\left|M_{++}\right|^{2}=-e_{f}^{2} g^{2} g_{s}^{2} \frac{C_{F}}{N_{c}} \frac{8 \epsilon}{t u}\left[Q^{2} s+(t+u)^{2}\right],
$$

which is proportional to $\epsilon$ and vanishes in the limit $\epsilon \rightarrow 0$. Since at the Born level there are no $\frac{1}{\epsilon}$ singularities that might combine with this term to yield a finite contribution, in the limit $\epsilon \rightarrow 0$,

$$
\left|M_{++}\right|^{2}=0 \text { and } \Delta|M|^{2}=-|\bar{M}|^{2} .
$$

For the $q+\bar{q} \rightarrow \gamma^{*}+g$ subprocess the condition that $\left|M_{++}\right|^{2}=0$ means that the quark helicity is maintained (does not flip) in the collision. The incoming quark line with helicity $\pm \frac{1}{2}$ turns around and becomes an incoming antiquark with helicity $\mp \frac{1}{2}$. 
We refer to this as "helicity conservation". We see that at the Born level helicity is conserved in the limit $\epsilon \rightarrow 0$.

In $N=4-2 \epsilon$ dimensions the differential cross section is related to the $2 \rightarrow 2$ invariant amplitude according to

$$
s \frac{d \sigma}{d t}(s, t, h)=\frac{1}{16 \pi s}\left(\frac{4 \pi s}{t u}\right)^{\epsilon} \frac{1}{\Gamma(1-\epsilon)}|M(h)|^{2},
$$

which can be written as

$$
s \frac{d \sigma}{d t}(s, t, h)=(1+h) s \frac{d \bar{\sigma}}{d t}(s, t)-h s \frac{d \sigma_{++}}{d t}(s, t)
$$

or as

$$
s \frac{d \sigma}{d t}(s, t, h)=s \frac{d \bar{\sigma}}{d t}(s, t)-h s \frac{d \sigma_{L L}}{d t}(s, t),
$$

where $s d \bar{\sigma} / d t$ is the unpolarized cross section and

$$
s \frac{d \sigma_{L L}}{d t}=\frac{1}{2}\left(s \frac{d \sigma_{++}}{d t}-s \frac{d \sigma_{+-}}{d t}\right) .
$$

At the Born level we have,

$$
\begin{aligned}
& s \frac{d \bar{\sigma}}{d t}(s, t)=e_{f}^{2} K_{2} \frac{\alpha_{s}}{s} T_{B}\left(Q^{2}, u, t\right), \\
& s \frac{d \sigma_{++}}{d t}(s, t)=-e_{f}^{2} K_{2} \frac{\alpha_{s}}{s} \epsilon A_{B}\left(Q^{2}, u, t\right),
\end{aligned}
$$

where

$$
\begin{aligned}
& T_{B}\left(Q^{2}, u, t\right)=\frac{2}{t u}\left[(1-\epsilon)\left(2 Q^{2} s+(1-\epsilon)\left(t^{2}+u^{2}\right)-2 \epsilon t u\right)\right] \\
& =2(1-\epsilon)\left[(1-\epsilon)\left(\frac{u}{t}+\frac{t}{u}\right)+\frac{2 Q^{2}\left(Q^{2}-u-t\right)}{u t}-2 \epsilon\right]
\end{aligned}
$$

and

$$
A_{B}\left(Q^{2}, u, t\right)=\frac{8}{t u}\left[Q^{2} s+(t+u)^{2}\right]
$$

where $K_{2}$ is defined by

$$
K_{2}=\pi \alpha \frac{C_{F}}{N_{c}} \frac{1}{\Gamma(1-\epsilon)}\left(\frac{4 \pi \mu^{2}}{Q^{2}}\right)^{\epsilon}\left(\frac{s Q^{2}}{t u}\right)^{\epsilon},
$$

where we have rescaled, $\alpha_{s} \rightarrow \alpha_{s}\left(\mu^{2}\right)^{\epsilon}$, so that it remains dimensionless in $N=4-2 \epsilon$ dimensions. At this order of perturbation theory, we have

$$
s \frac{d \sigma}{d t}(s, t, h)=e_{f}^{2} K_{2} \frac{\alpha_{s}}{s}\left[(1+h) T_{B}\left(Q^{2}, u, t\right)+h \epsilon A_{B}\left(Q^{2}, u, t\right)\right] .
$$


As we saw earlier, in the limit $\epsilon \rightarrow 0$ helicity is conserved so that

$$
s \frac{d \sigma}{d t}(s, t, h)=(1+h) s \frac{d \bar{\sigma}}{d t}(s, t)
$$

which implies that

$$
s \frac{d \sigma_{++}}{d t}(s, t)=0 \text { and } s \frac{d \sigma_{L L}}{d t}(s, t)=-s \frac{d \bar{\sigma}}{d t}(s, t) .
$$

To connect with the notation of ref. [3], we note that

$$
K_{2} T_{B}\left(Q^{2}, u, t\right)=K T_{0}\left(Q^{2}, u, t\right)
$$

where $K$ and $T_{0}$ are define in ref. [3] as

$$
K=2 \pi \alpha \frac{C_{F}}{N_{c}} \frac{(1-\epsilon)}{\Gamma(1-\epsilon)}\left(\frac{4 \pi \mu^{2}}{Q^{2}}\right)^{\epsilon}\left(\frac{s Q^{2}}{t u}\right)^{\epsilon}
$$

and

$$
T_{0}\left(Q^{2}, u, t\right)=\left[(1-\epsilon)\left(\frac{u}{t}+\frac{t}{u}\right)+\frac{2 Q^{2}\left(Q^{2}-u-t\right)}{u t}-2 \epsilon\right]
$$

\section{Virtual diagram contribution}

The list of the diagrams with virtual corrections contributing to the non-singlet sector of Drell-Yan is given in Fig. 4. We have omitted all the self-energy insertions of quarks and gluons and the ghost contributions.

Our calculations are performed in the $\overline{M S}$ scheme using dimensional regularization to regulate both the ultraviolet and the infrared singularities. We remove the ultraviolet singularities in the relevant subdiagrams by off shell regularization. Then by sending on shell the initial state quarks and the final state gluon, we encounter singularities in the form of double poles and single poles in $\epsilon=2-n / 2$. The one-loop order result for the cross section for the process $q+\bar{q} \rightarrow \gamma^{*}+g$ is given by

$$
\begin{aligned}
& s \frac{d \sigma^{\text {virtual }}}{d t d u}(s, t, u, h)= \\
& e_{f}^{2} K_{2} \frac{\alpha_{s}}{s} \delta\left(s+t+u-Q^{2}\right)\left\{( ( 1 + h ) T _ { B } + h \epsilon A _ { B } ) \left[1-\frac{\alpha_{s}}{2 \pi} \frac{\Gamma(1-\epsilon)}{\Gamma(1-2 \epsilon)}\left(\frac{4 \pi \mu^{2}}{Q^{2}}\right)^{\epsilon}\right.\right. \\
& \left.\times\left(\frac{2 C_{F}+N_{C}}{\epsilon^{2}}+\frac{1}{\epsilon}\left(3 C_{F}-2 C_{F} \ln \frac{s}{Q^{2}}+\frac{11}{6} N_{C}+N_{C} \ln \frac{s Q^{2}}{u t}-\frac{1}{3} N_{F}\right)\right)\right] \\
& +\frac{\alpha}{2 \pi}(1+h)\left[\pi^{2}\left(4 C_{F}+N_{C}\right) \frac{2 Q^{2} s+t^{2}+u^{2}}{3 t u}-2\left(2 C_{F}-N_{C}\right) \frac{Q^{2}\left(t^{2}+u^{2}\right)}{t u(t+u)}\right.
\end{aligned}
$$




$$
\begin{aligned}
& -2 C_{F}\left(\frac{8\left(2 Q^{2} s+t^{2}+u^{2}\right)}{t u}-\frac{Q^{4} s(t+u)}{t u\left(Q^{2}-u\right)\left(Q^{2}-t\right)}-\frac{t^{2}+u^{2}}{\left(Q^{2}-u\right)\left(Q^{2}-t\right)}\right) \\
& -2\left(L i_{2}\left(\frac{t}{t-Q^{2}}\right)+\frac{1}{2} \ln ^{2}\left(1-\frac{Q^{2}}{t}\right)\right)\left(N_{C} \frac{2 s+t}{u}+2 C_{F} \frac{s^{2}+(s+u)^{2}}{t u}\right) \\
& -2\left(L i_{2}\left(\frac{u}{u-Q^{2}}\right)+\frac{1}{2} \ln ^{2}\left(1-\frac{Q^{2}}{u}\right)\right)\left(N_{C} \frac{2 s+u}{t}+2 C_{F} \frac{s^{2}+(s+t)^{2}}{t u}\right) \\
& +2\left(2 C_{F}-N_{C}\right)\left(L i_{2}\left(-\frac{t+u}{s}\right) \frac{2 Q^{2} s+u^{2}+t^{2}+2 s^{2}}{t u}\right. \\
& +\left(2 \ln \left(\frac{s}{Q^{2}}\right) \frac{Q^{4}-(t+u)^{2}}{(t+u)^{2}}+\ln ^{2}\left(\frac{s}{Q^{2}}\right) \frac{s^{2}}{t u}\right) \\
& -\left(\ln \left(\frac{|t|}{Q^{2}}\right) \ln \left(\frac{s}{Q^{2}}\right)-\frac{1}{2} \ln ^{2}\left(\frac{|t|}{Q^{2}}\right)\right) \frac{s^{2}+(s+u)^{2}}{t u} \\
& \left.-\left(\ln \left(\frac{s}{Q^{2}}\right) \ln \left(\frac{|u|}{Q^{2}}\right)-\frac{1}{2} \ln ^{2}\left(\frac{|u|}{Q^{2}}\right)\right) \frac{s^{2}+(s+t)^{2}}{t u}\right) \\
& +2 \ln \left(\frac{|u|}{Q^{2}}\right)\left(C_{F} \frac{4 Q^{2} s-2 s u+t u}{\left(Q^{2}-u\right)^{2}}+N_{C} \frac{u}{Q^{2}-u}\right) \\
& +2 \ln \left(\frac{|t|}{Q^{2}}\right)\left(C_{F} \frac{4 Q^{2} s-2 s t+t u}{\left(Q^{2}-t\right)^{2}}+N_{C} \frac{t}{Q^{2}-t}\right) \\
& \left.\left.-2 \ln \left(\frac{|t|}{Q^{2}}\right) \ln \left(\frac{|u|}{Q^{2}}\right) N_{C} \frac{2 Q^{2} s+t^{2}+u^{2}}{t u}\right]\right\}
\end{aligned}
$$

where $h=h_{1} h_{2}$. The quantities $T_{B}$ and $A_{B}$ appear in at the Born level (22) and are given by equations (19) and (20), respectively. Notice that the double poles and single pole structure (in the $h \rightarrow 0$ limit) automatically reproduces the singularities of the virtual contributions of ref. [3]. In particular, the double poles in $\epsilon$ correctly multiply the two-to-two Born contribution $T_{B}$, which is the Born level unpolarized result. Furthermore, it is possible to show [12] that the structure of the virtual contributions presented here correctly factorize, after adding the real contributions.

The presence of the $h \epsilon A_{B}$ term in (28) implies that in the t'Hooft-Veltman $[9,10]$ scheme the virtual corrections by themselves do not conserve helicity (i.e. they do not satisfy the equation (23)). In particular, the finite part of $d \sigma_{++} / d t$ in the limit $\epsilon \rightarrow 0$ for the virtual corrections is given by

$$
\begin{aligned}
& s \frac{d \sigma_{++}^{\text {virtual }}}{d t}(s, t)= \\
& -e_{f}^{2} K_{2} \frac{\alpha_{s}^{2}}{2 \pi s} A_{B}(s, t)\left(3 C_{F}-2 C_{F} \ln \frac{s}{Q^{2}}+\frac{11}{6} N_{C}+N_{C} \ln \frac{s Q^{2}}{u t}-\frac{1}{3} N_{F}\right),
\end{aligned}
$$

where $s+t+u=Q^{2}$. In some regularization schemes, such as those enforcing an anticommuting $\gamma_{5}$ in $n$ dimensions, helicity would be conserved and equation 
(13) maintained throughout. In the t'Hooft-Veltman $[9,10]$ scheme helicity is not manifestly conserved. However, we expect that the non-singlet real contributions, $s d \sigma_{++}^{\text {real }} / d t$, will cancel the virtual contribution in (29) leaving a final result that conserves helicity and maintains equation (23). Work on the helicity dependent real corrections to order $\alpha_{s}^{2}$ is in progress [12]. A study of the transversity distribution to $O\left(\alpha_{s}^{2}\right)$ is also forthcoming [13].

\section{Conclusions}

We have presented the parton-level analytical results for the next-to-leading order non-singlet virtual corrections to the Drell-Yan differential cross-section. The dependence of the differential cross section on the helicity of the initial state partons is shown explicitly (the spins of the final state partons are summed). Although the calculation is very involved due to the presence of chiral projectors in the initial state, the result is quite compact and has been presented in a form from which cancelation of the infrared, collinear and infrared plus collinear singularities is evident. Both the polarized initial state and the unpolarized cross sections can be obtained from our result. Our unpolarized cross section agrees with the previous result of Ellis, Martinelli and Petronzio [3] in the non-singlet sector. Our calculation is a first step toward the extension of the classical $O\left(\alpha_{s}^{2}\right)$ Drell-Yan result to the case of longitudinal polarized beams. A more detailed discussion of our results and of the methods developed by us in the analysis of the virtual corrections will be presented elsewhere [12], where we will provide a complete analysis of the initial state helicity dependence in the non-singlet sector.

\section{Acknowledgments}

S. C. and C.C. thank Alan White and the Theory Group at Argonne for hospitality. C.C. thanks P. Nason and the Theory Division at Cern and G. Marchesini and Y. Dokshitzer at the Univ. of Milan for hospitality. We also thank L.E. Gordon and J. Elwood for useful discussions.

\section{References}

[1] G.P. Ramsey, Probing Nucleon Spin Structure hep-ph 9702227, ANL-HEP-PR 96-103.

[2] C. Bourrely, J. Soffer, F.M. Renard and P. Taxil, Phys. Rep. 177, 320 (1989).

[3] R. K. Ellis, G. Martinelli and R. Petronzio, Nucl. Phys. B211 (1983) 106 
[4] C. Corianò and L. E. Gordon, Nucl. Phys. B469 (1996) 202, Phys. Rev. D54 781 (1986).

[5] R Mertig and W. L.R. Mertig, M. Bohm, A. Denner Comput. Phys. Commun. 64 (1991) 345-359

[6] P. Ratcliffe, Nucl. Phys. B223, 45 (1983).

[7] G. Passarino and M. Veltman, Nucl. Phys. B160 (1979) 151

[8] R. L. Jaffe hep-ph/9602236

[9] G. t'Hooft and M. Veltman, Nucl. Phys. B44, 189 (1972).

[10] P. Bretenlohner and D. Maison, Comm. Math. Phys. 52 (1977) 11;

[11] R. J. Gonsalves, Phys. Rev. D28 (1983) 1542; G. Kramer and B. Lampe, Z. Phys. C34 (1987) 497; T. Matsuura and W. L. Van Neerven, Z. Phys. C38 (1988) 623.

[12] S. Chang, C. Corianò, R. D. Field and L. E. Gordon, in preparation.

[13] S. Chang. C. Corianò and J. Elwood, work in progress. 
Figures

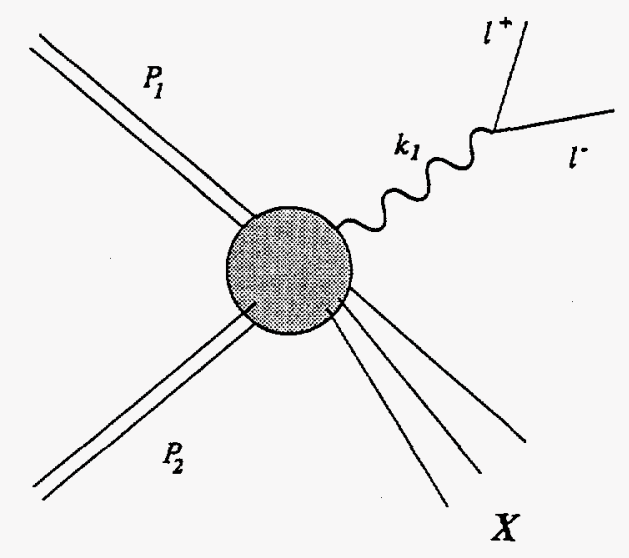

Figure 1: The Drell-Yan process
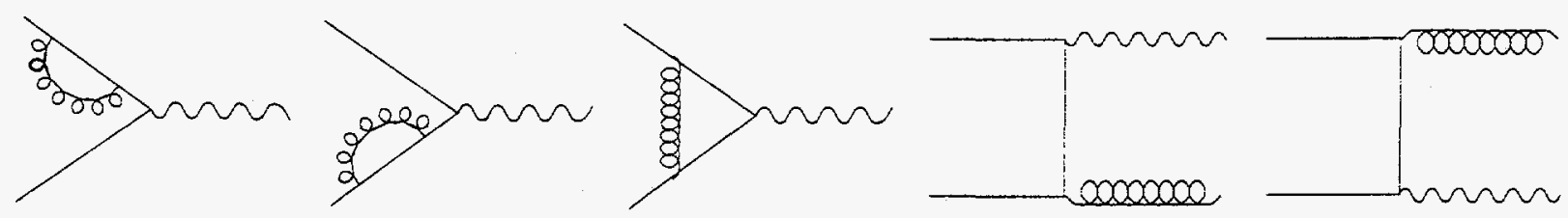

Figure 2: Radiative corrections of order $\alpha_{s}$
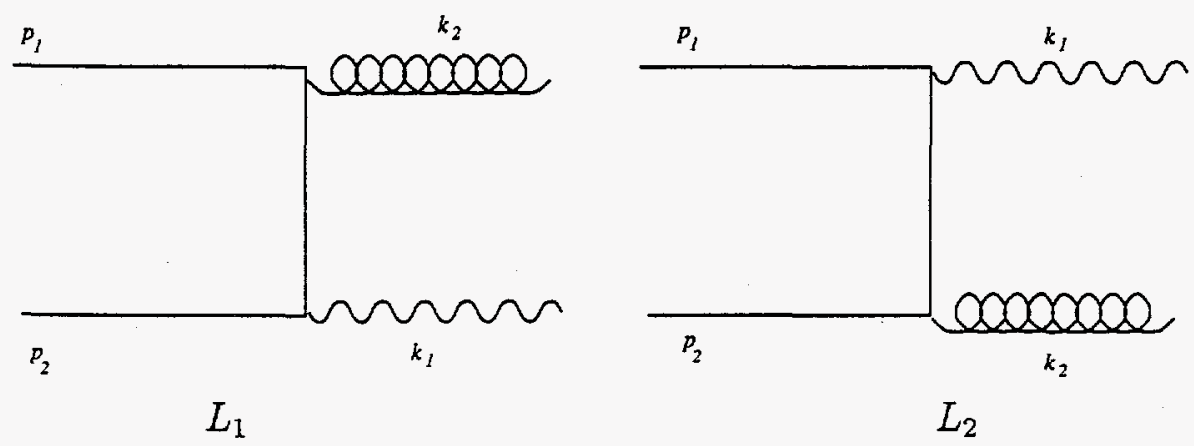

Figure 3: The Born diagrams 

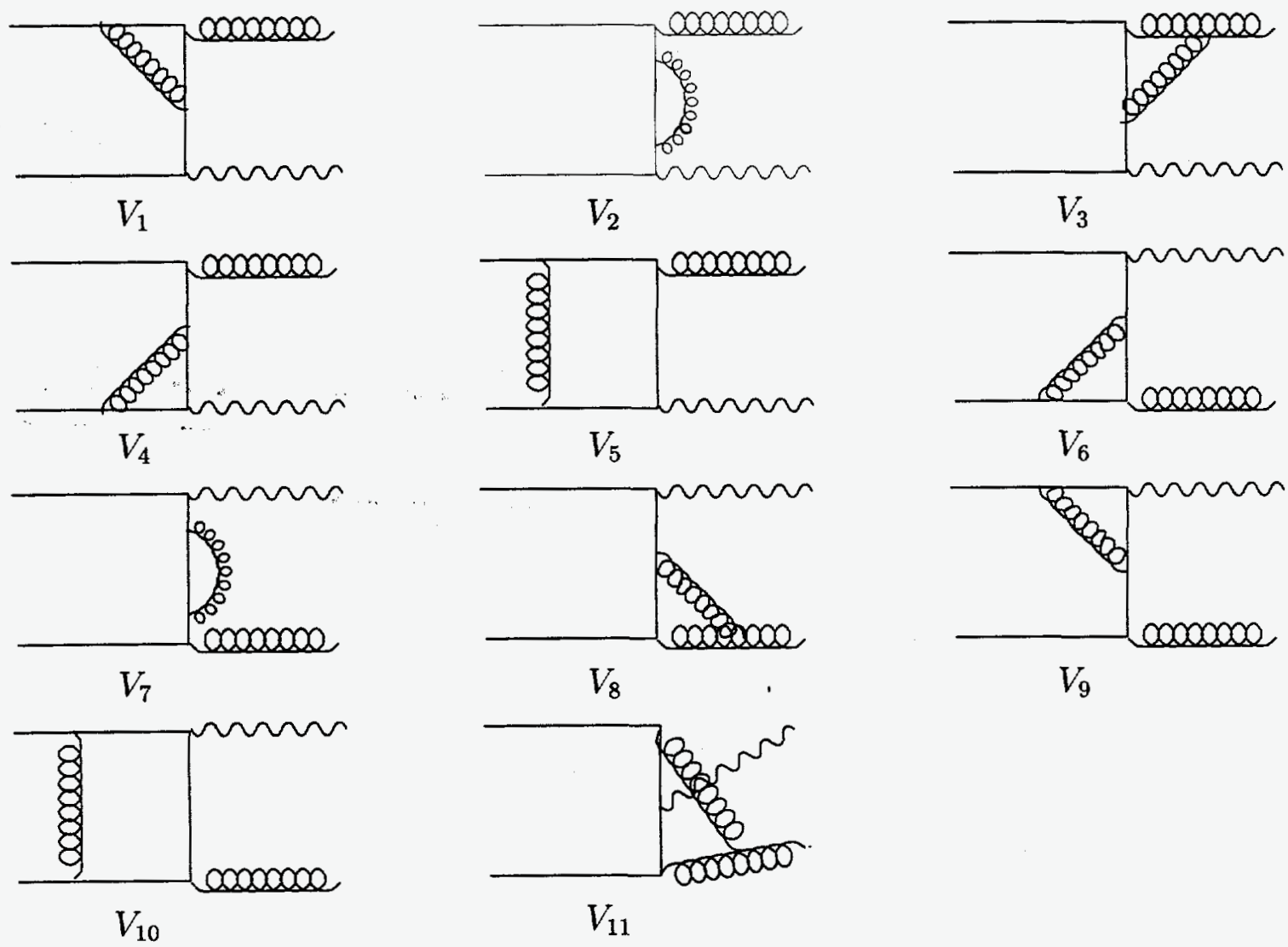

Figure 4: One-Loop contributions 